\title{
Preparation and Properties of A Novel Y-type Nonlinear Optical Polyester with Dioxybenzylidenecyanoacetate Groups
}

\author{
Ga-Young Lee, Dong-Seon Won, Han-Na Jang, Hyo Jin No, and Ju-Yeon Lee* \\ Institute of Basic Science, Department of Chemistru, Inje Cniversitv, Gimhae 621-749, Korea \\ E-mail: chemljwainje ack' \\ Recerved February 17, 2009, Accepted Harch 20,2009
}

\begin{abstract}
Methyl 2,4-di-(2'-hydroxyethoxy)benzy lidenecyanoacetate (3) was prepared and polymerized with terephthaloyl chloride to yield a novel Y-ty'pe polyester 4 containing 2,4-dioxybenzylidenecyanoacetate groups as NLOchromophores, which constituted parts of the polymer backbone. The resulting polymer $t$ is soluble in conmon organic solvents such as acetone and $F, N$-dimethylformamide. Polymer 4 showed themal stability up to $280^{\circ} \mathrm{C}$ in thermogravimetric analysis with glass-transition temperature obtained from differential scanning calorimetry near $108^{\circ} \mathrm{C}$. The second harmonic generation ( $\mathrm{SHG}$ ) coefficient $\left(d_{33}\right)$ of poled polymer films at the 1064 nm fundamental wavelength was around $3.54 \times 10^{\circ}$ esu. The dipole alignment exhibited a thermal stability up to near $T_{z}$ and no significant $\mathrm{SHG}$ decay was observed below $100^{\circ} \mathrm{C}$ due to the partial main-chain character of polymer structure, which is acceptable for NLO device applications.
\end{abstract}

Key Words: Nonlinear optics, Polyester. AFM. Second harnonic generation, Dipole alignment

\section{Introduction}

Recently functional polymers of nonlinear optical (NLO) activity are studied extensively because of their potential applications in the field of electro-optic devices..$^{1-4}$ A potential NLO polymer has to be mechanically very strong and thermally stable with a high glass-transition temperature $\left(T_{g}\right)$. In the developments of NLO polymers for electro-optic device applications, stabilization of electrically induced dipole alignment is one of important criteria: in this context. two approaches have been proposed to minimize the randomization of dipole alignment namely the use cross-linking method ${ }^{5-k}$ and the utilization of high $T_{z}$ polymers such as polyimides. ${ }^{9}$ Various polyesters with NLO-chromophores in the main chain ${ }^{110}$ or in side chain ${ }^{11}$ have been prepared and their properties studied. Main-chain NLO polymers generally have good thermal stability of dipole alignments. but they often do not dissolve in organic solvents, and their intractability make them unusable to fabricate stable films. Side-chain NLO polymers have the advantages such as good solubility and homogeneity relative to the main-chain systems. but they often suffer from poor stability of dipole alignments at high temperatures. Recently we have prepared novel polyesters containing dioxynitrostil-

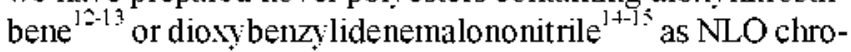
mophores. The resulting polymers exhibited enhanced thermal stability of second harmonic generation (SHG), which stemmed from the stabilization of dipole alignment of the NLO chromophores.

In this work we prepared a novel polyester containing 2.4-dioxybenzylidenecyanoacetate groups as NLO-chromophores. We selected the latter because they have large dipole moments and are rather easy to synthesize. Furthermore, 2.4dioxybenzylidenecyanoacetate groups constitute novel Y-type NLO polyesters (Fig. Ic), and these Y-type NLO polyesters have not been reported in the literature. Thus. we synthesized NLO polyester in which the pendant NLO chromophores are (a)

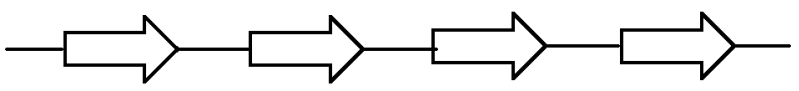

(b)

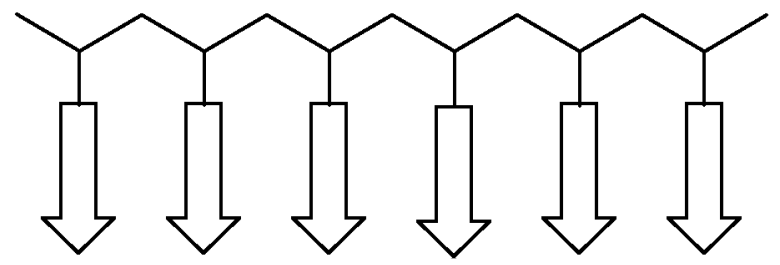

(c)

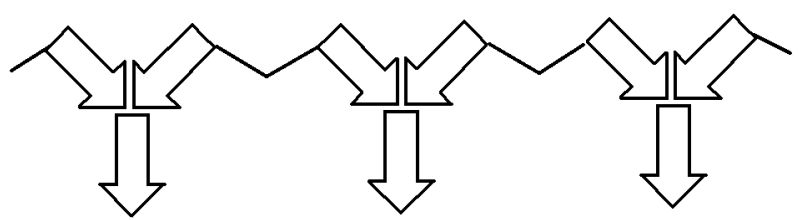

Figure 1. Schematic structures of NLO polymers with chromophores located (a) main-chain, (b) side-chain, and (c) main/side chain (Y-type).

parts of the polymer backbones. This mid-type NLO poly mer is expected to have the advantages of both main-chain and side-chain NLO polymers namely stable dipole alignment and good solubility. After confirming the structure of the resulting polymer we investigated its properties such as solubility. $T_{\mathrm{s}}$. thermal stability. SHG activity and relaxation of dipole alignment.

\section{Results and Discussion}

Synthesis of Polymer 4. 2,4-Di-(2'-vinyloxyethoxy)benzaldelyde (1) was prepared by the reaction of 2-chloroethyl vinyl ether with 2.4-dihydroxybenaldehyde. Methyl 2.4-di(2'-viny loxyethosy)benzy lidenecyanoacetate (2) was prepared by the condensation reaction of 1 with metlyl cyanoacetate. Compound $\mathbf{2}$ was hyddrolyzed to yield compound $\mathbf{3}$. Diol $\mathbf{3}$ was 

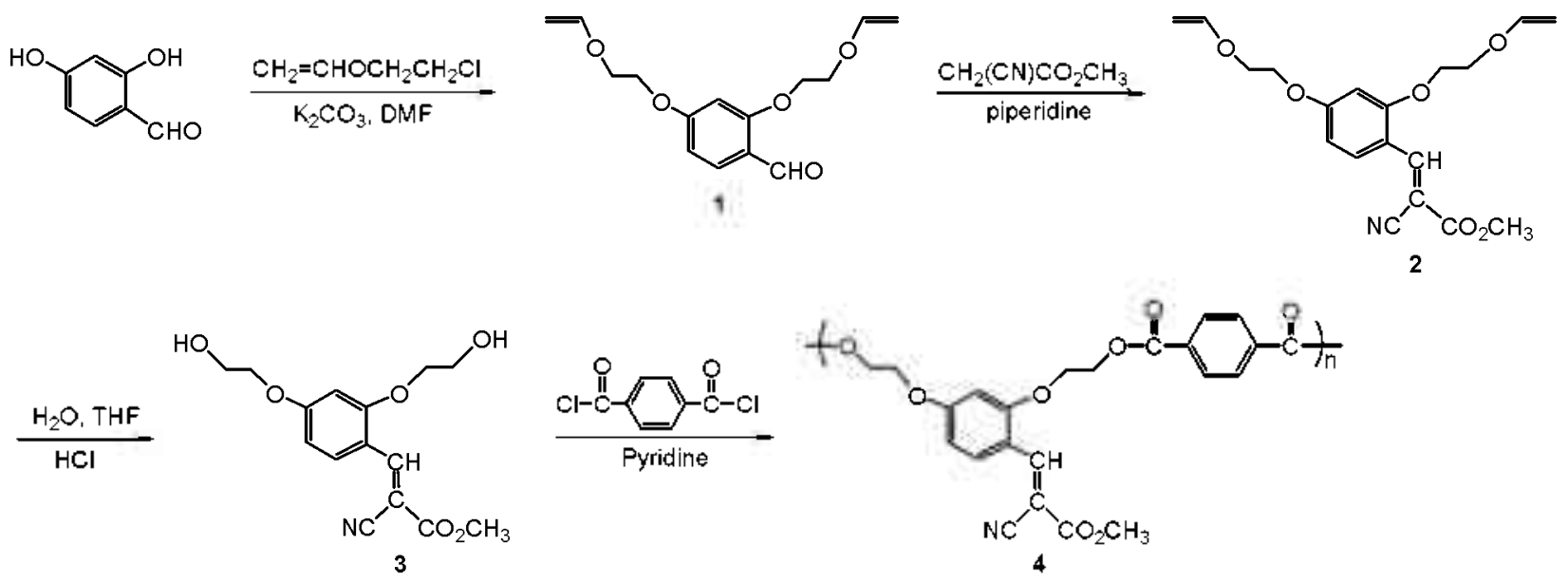

Scheme 1. Synthetic scheme and structure of polymer 4

condensed with terephthaloyl chloride (TPC) in a dry DMF solvent to yield polyester 4 containing 2.4-dioxybenzylidenecyanoacetate groups as NLO-chromophores. The synthetic route for polymer 4 is presented in Scheme 1 . The polymerization yield was around $91 \%$. The chemical stnicture of the resulting polymer was confirmed by ${ }^{1} \mathrm{H}$ NMR, IR spectra, and elemental analysis. Elemental analysis results fit the polymer structures. ${ }^{1} \mathrm{H}$ NMR spectrum of the polymer showed a signal broadening due to polymerization. but the chemical shifts are consistent with the proposed polymer structure. The IR spectrum of the polymer sample shows a strong carbonyl peak near $1722 \mathrm{~cm}^{-1}$ indicating the presence of ester bond. The same polymer sample also showed a strong ritrile peak near $2220 \mathrm{~cm}^{-1}$. These results are consistent with the proposed polymer structure. indicating that the NLO-chromophore remained intact during the polymerization. The molecular weight was determined by GPC using polystyrene as the standard and tetrallydrofuran (THF) as the eluent. The number average molecular weight $\left(a L_{n}\right)$ of the polymer 4 was 18.200 $\left(M_{w} M_{n}=1.91\right)$. The structural feature of this poly mer is that it has pendant NLO chromophores. which are parts of the polymer main chains. Thus the resulting polymer 4 is a mid type of side chain- and main-chain NLO polymer. and is expected to have both their merits. The polymer 4 is soluble in common solvents such as acetone. DMF. and DMSO, but is not soluble in methanol and diethyl ether. The inherent viscosity was around $0.30 \mathrm{dL} / \mathrm{g}$. Polymer 4 showed strong absorption near $364 \mathrm{~nm}$ by the NLO-chromophore 2.4-dioxybenzylidenecyanoacetate. We now have well defined polyester (4) and investigate its properties.

Thermal Property. The thermal behavior of the polymer was investigated by TGA and DSC to determine the thermal degradation pattern and glass transition temperature. The results are summarized in Table 1. DSC thermogram of polymer 4 is presented in Fig. 2. Polymer 4 showed a thermal stability up to $280^{\circ} \mathrm{C}$ according to its TGA thermogram. The $T_{g}$ value of the polymer 4 measured by DSC was around 108 ${ }^{\circ} \mathrm{C}$. This $T_{\mathrm{g}}$ value is somewhat higher than that of the polyester containing 2.4-dioxybenzylidenemalononitrile. ${ }^{15}$ The TGA and DSC studies showed that the decomposition temperature of the polyester 4 was higher than the corresponding $T_{g}$. This
Table 1. Thermal Properties of Polymer 4

\begin{tabular}{|c|c|c|c|c|}
\hline \multirow{2}{*}{ Polymet } & \multirow{2}{*}{$\begin{array}{c}\mathrm{T}_{\mathrm{z}}{ }^{a} \\
(\mathrm{C})\end{array}$} & \multicolumn{2}{|c|}{ Degradation temp $\left({ }^{\circ} \mathrm{C}\right)^{b}$} & \multirow{2}{*}{$\begin{array}{l}\text { Residue at } \\
800^{\circ} \mathrm{C}(\%)\end{array}$} \\
\hline & & 5 ut $\%$ - loss 20 ut $\%$-loss & $40 \mathrm{w} \%-10 \mathrm{ss}$ & \\
\hline 4 & 108 & 337 & 369 & 14.1 \\
\hline
\end{tabular}

Detenmined from DSC curves measured on a TA 2920 difterential scanning calorimeter with a heating rate of $10^{\circ} \mathrm{C}$ min under nitrogen atmosphere. Detemined from TGA curves measured on a TA Q50 thermogravimetric analyzer with a heating rate of $10^{\circ} \mathrm{C}$ min under nitrogen atmosphere.

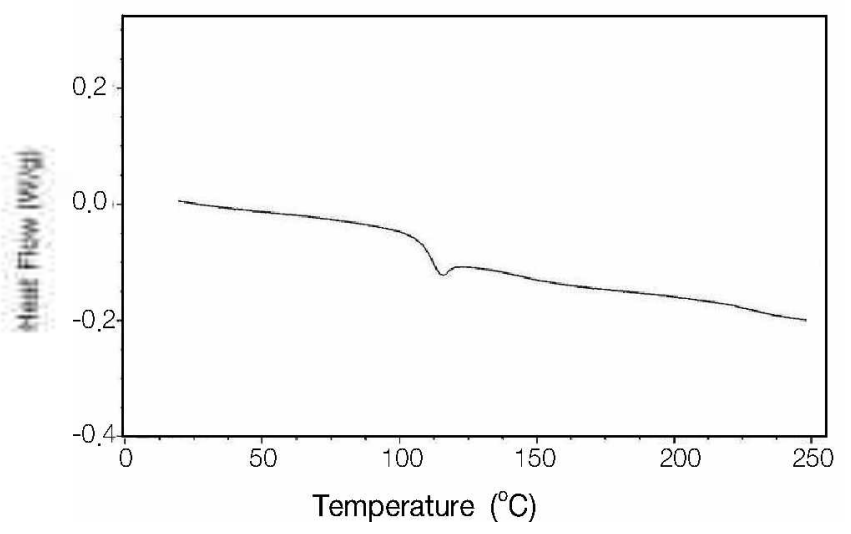

Figure 2. DSC themogram of polymer 4 at a heating rate of $10^{\circ} \mathrm{C} /$ min under nitrogen.

indicates that high-temperature poling for a short term is feasible without damaging the NLO chromophore.

Nonlinear Optical Property. The NLO properties of polymer 4 were studied by the SHG method. To induce noncentrosymmetric polar order, the spin-coated polymer film was corona-poled. As the temperature was raised to $5-10^{\circ} \mathrm{C}$ higher than $T_{g}, 6.5 \mathrm{kV}$ of corona voltage was applied and kept at that temperature for $30 \mathrm{~min}$. The poling was confirmed by UV-vis spectrum. Polymer 4 showed strong absorption near $364 \mathrm{~nm}$ by the NLO-cliromophore 2.4-dioxybenzylidenecyanoacetate group. After electric poling the dipole moments of the NLOchromophores were aligned and the UV-vis spectrum of polymer 4 exhibited a decrease in absorption due to birefringence. From the absorbance change, the order parameter 
(ii)

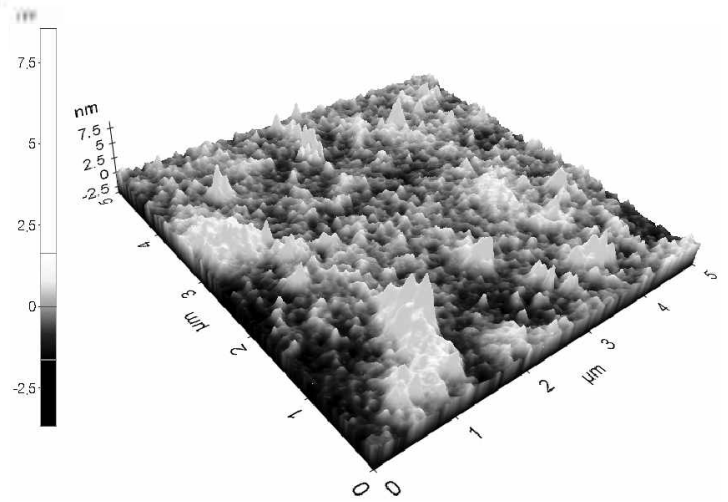

(b)

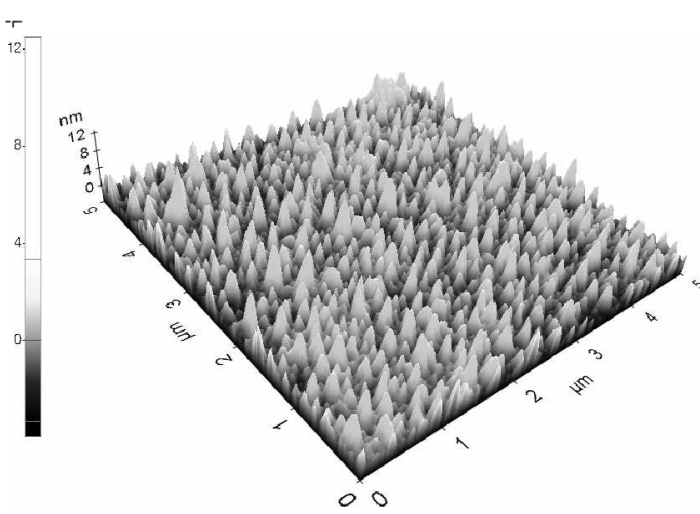

Figure 3. AFM images of spin-coated tilm of polymer 4: (a) before corona-poling: (b) after corona-poling.

of the poled film could be estimated. which is related to the poling efficiency. The estimated order parameter value $\Phi$ was equal to 0.13 for polymer $+\left(\Phi=1-A_{1} / A_{i c}\right.$ where $A_{i}=1.2612$ and $A_{l}=1.1001$ are the absorbances of the polymer film before and after poling. respectively). For the purpose of investigating surface morphology of polymer film. domain structures of NLO-chromophores for the thin-film sample were obtained using atomic force microscopy (AFM). Fig. 3 shows AFM scans of the spin-coated film before and after poling polymer $t$. AFM images show that the surface of the film sample is extremely flat and clean before poling (see Fig. 3a). However. this good quality film was dramatically changed after poling. resulting in numerous hills and valley's in the surface structure, which means that the NLO-chromophores are aligned the poling direction as shown in Fig. 3b.

The refractive index of the sample was measured by the optical transmission technique. ${ }^{16}$ The transmittance of thin film includes information on the thickness. refractive index and its extinction coefficient. Thus, we can determine those parameters by analyzing the transmittance. SHG measurements were performed at a fundamental wavelength of 1064 rum using a mode locked Nd-YAG laser. In order to determine the microscopic second-order susceptibility of the polymer. the angular SHG dependence was recorded. Fig. 4 shows the angular dependence of SHG signal in a poled polymer 4 . The SHG values were compared with those obtained from a Y-cut quartz plate. To calculate the $d_{3 /}$ and $d_{33}$ values, both $s$-polarized and $p$-polarized IR laser were directed to the sample and

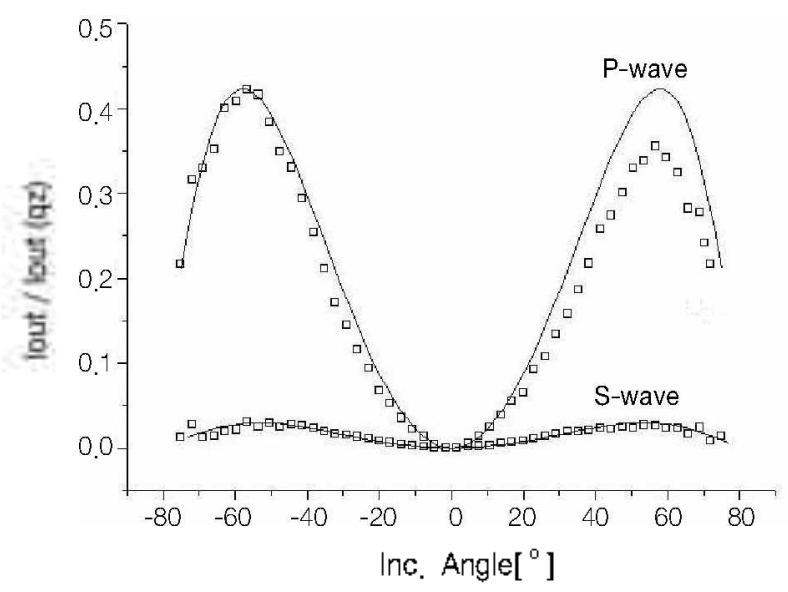

Figure 4. Angular dependence of $\mathrm{SHG}$ signal in a poled film of polymer 4 .

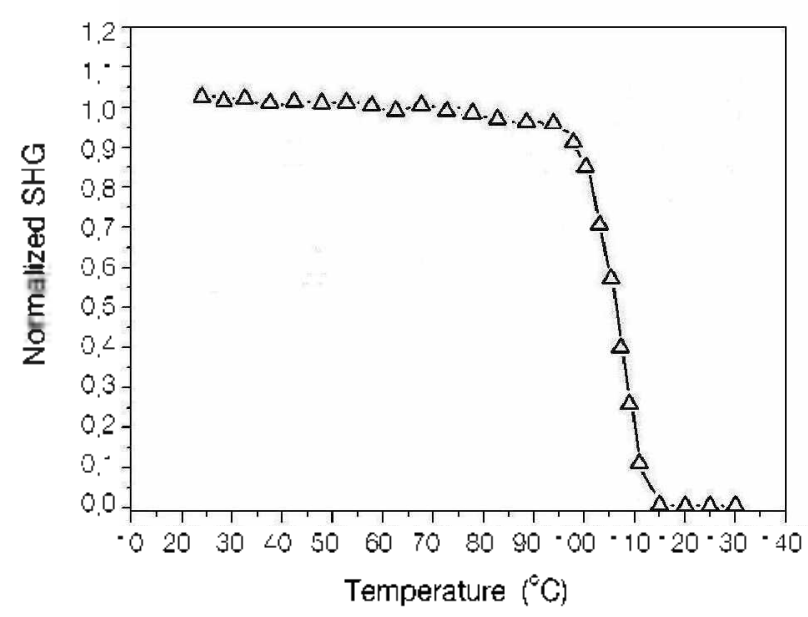

Figure 5. Normalized SHG signal of polymer 4 as a function of temperature at a heating rate of $3.5^{\circ} \mathrm{C} / \mathrm{min}$.

Table 2. Nonlinear Optical Properties of Polymer 4

\begin{tabular}{|c|c|c|c|c|c|c|}
\hline Polymer & $\begin{array}{l}\lambda_{\max }^{a} \\
(\text { inin) }\end{array}$ & $\begin{array}{c}d_{33}^{b} \\
(\mathrm{esu})\end{array}$ & $\Phi$ & $\begin{array}{l}\text { film } \\
\text { thickness } \\
\text { ( } \mu \mathrm{m})\end{array}$ & $\begin{array}{c}d_{31}^{b} \\
(\mathrm{esul})\end{array}$ & $n$ \\
\hline 4 & 364 & $\begin{array}{c}(3.54 \pm 0.09) \\
\times 10^{9}\end{array}$ & 0.13 & 0.52 & $\begin{array}{c}(1.26 \pm 0.06) \\
\times 10^{9}\end{array}$ & $\begin{array}{l}\mathrm{n}_{1}=1.60 \\
\mathrm{n}_{2}=1.66\end{array}$ \\
\hline
\end{tabular}

"Polymer film after corona poling. "SHG coefficients $\left(d_{3}\right)$ were derived from the analysis of measured Maker-finges. " 1- $A_{1}: A_{0}$. where $A_{n}$ and $A_{1}$ are the absorbances of the polymer film before and after corona poling. respectivelv. "Film thickness was determined by. the optical transmission technique. ${ }^{\text {i6 }}$

recorded. Nonlinear optical properties of polymer $t$ are summarized in Table 2 . SHG coefficients $\left(d_{3}\right)$ were derived from the analysis of measured Maker-fringes with the Pascal fitting progran according to the literature procedure. ${ }^{17}$ The values of $d_{33}$ and $d_{3,3}$ for polymer 4 were $1.26 \times 10^{-9}$ esu and $3.54 \times 10^{9}$ esu, respectively. These values are somewhat smaller than those for the polyester containing 2.4-dioxybenzylidenemalononitrile group. " probably because of the weaker electron-attracting tendency of carbosylate than cyano 
group. Since the second hamonic wavelength was at $532 \mathrm{~mm}$, which is not in the absorptive region of the resulting polymer. there was not resonant contribution to this $d_{33}$ value.

To evaluate the high-temperature stability of the polymers. we studied the temporal stability of the SHG signal. In Fig. 5. we present the dynamic thermal stability study of the NLO activity of the film 4 . To investigate the real time NLO decay of the SHG signal of the poled polymer film as a function of temperature. in situ SHG measurements were performed at a heating rate of $3.5^{\circ} \mathrm{C} / \mathrm{min}$ from 30 to $150^{\circ} \mathrm{C}$. The poly mer film exhibited a thermal stability up to near $T_{g}$ and no significant SHG decay was obsened below $100^{\circ} \mathrm{C}$. as shown in Fig. 5 . In general. side-chain NLO polymers lose thermal stability of dipole alignment below $T_{g}$. Stabilization of dipole alignment is a characteristic of main chain NLO polymers. The high thermal stability of polymer 4 was due to the stabilization of dipole alignment of NLO chromophore, which stemmed from the partial main chain character of the polymer structure. Thus. we obtained a new type of NLO polyester having the advantages of both main-chain and side-chain NLO polymers: stabilization of dipole alignment and good solubility.

\section{Conclusions}

We synthesized a novel Y-type polyester 4 with pendant NLO chromophores. which are parts of the polymer main chains. This mid-type NLO polyester is soluble in common organic solvents. The resulting polymer + showed a thermal stability up to $280^{\circ} \mathrm{C}$ from TGA thermogram with $T_{g}$ value near $108^{\circ} \mathrm{C}$. The SHG coefficient $\left(d_{33}\right)$ of corona-poled polymer film was $3.54 \times 10^{-5}$ estu. The striking feature of this polymer is that it exhibit SHG stability up to near $T_{g}$ and no significant SHG decay was observed below $100^{\circ} \mathrm{C}$. This high thermal stability of optical nonlinearity stemmed from the stabilization of dipole alignment of the NLO-chromophore. which constituted a part of the poly mer backbone. We are now in the process of extending the polymerization system to the synthesis of other type of NLO polymers and the results will be reported elsewhere.

\section{Experimental Section}

Materials. The reagent grade chemicals were purchased from Aldrich and purified by either distillation or recrystallization before use. 2.4-Dihydroxybenzaldehyde and 2chloroethyl vinyl ether were used as received. Terephthaloyl chloride (TPC) was purified by sublimation under vacuum. Methỵl cyanoacetate was distilled under reduced pressure. Piperidine was treated with potassium hydroxide and then distilled over barium oxide to remove trace amounts of water. DMF was purified by drying with anhydrous calcium sulfate. followed by distillation under reduced pressure.

Measurements. IR spectra were taken on a Shimadzı FT IR-8201PC infrared spectrophotometer. ${ }^{\text {l }} \mathrm{H}$ NMR spectra were obtained on a Varian $300 \mathrm{MHz}$ NMR spectrometer. UV-Vis absorption spectra were measured on a Shimadzu UV-3100S spectrophotometer. Elemental analy ses were performed using a Perkin-Elmer $2400 \mathrm{CHN}$ elemental analyzer. $T_{\mathrm{g}}$ was mea- sured on a TA 2920 differential scanning calorimeter in a nitrogen atmosphere. TA Q50 thermogravimetric analyzer with a heating rate of $10^{\circ} \mathrm{C} / \mathrm{min}$ up to $800^{\circ} \mathrm{C}$ was used for the thermal degradation pattern of polymer under nitrogen. The number average molecular weight $\left(H_{n}\right)$ and weight average molecular weight $\left(M f_{n}\right)$ of the poly mer were estimated by gel permeation chromatographỵ (GPC) (columns sțragel HR5E$4 \mathrm{E}$ : solvent THF). Melting points were measured with a Buchi 530 melting point apparatus and are corrected. Viscosity values were obtained by using a Cannon-Fenske viscometer.

Film Preparation and SHG Measurement. The polymer film was prepared from a $10 \mathrm{wt} \%$ polymer solution in DMF deposited on an indium-tin oxide (ITO) covered glass. Prior to film casting. the polymer solution was filtered through $0.45 \mu \mathrm{m}$ Teflon ${ }^{5}$ membrane filter. The film was spin cast at room temperature in the range $1000-1200 \mathrm{rm}$. The films were dried for $12 \mathrm{~h}$ under vacuum at $60^{\circ} \mathrm{C}$. The alignment of the NLOchromophore of the polymers was carried out by corona poling method. The poling was performed in a wire-to plane geometry under in situ conditions. The discharging wire to plane distance was $10 \mathrm{~mm}$. As the temperature was raised gradually to $5-10^{\circ} \mathrm{C}$ higher than $T_{g} .6 .5 \mathrm{kV}$ of corona voltage was applied and kept that tenperature for $30 \mathrm{~min}$. The films were cooled to room temperature in the presence of the electric field. Finally, the electric field was removed. The refractive index of the sample was measured by the optical tra nsmission technique. ${ }^{16}$ SHG measurement was carried out one day after poling. A continuum PY6l mode-locked $\mathrm{Nd}$ : YAG laser $(\lambda=1064 \mathrm{~nm})$ with pulse width of $40 \mathrm{ps}$ and repetition rate of $10 \mathrm{~Hz}$ was used as the fundamental light source and Y-cut quartz was used as reference. A beam splitter and a photodiode were used to compensate for the intensity fluctuations of the fundamental bean $(106+\mathrm{nm})$. The polarity of the fundamental laser beam was adjusted using a half-wave plate before it hit the sample. The electric field vector of the incident beam was either parallel ( $p$-polarization) or perpendicular (s-polarization) to the plane of incidence. Only the $p$-polarized $\mathrm{SH}$ beam was made to enter a photomultiplier tube (PMT) by using a prișm and a SH pass filter. An analyzer was used to confirm the polarization direction of the $\mathrm{SH}$ signal. A poled polymer film was mounted on the rotator coupled to a step motor. The output signals from the photodiode and PMT were detected as a function of the incident angle. A 3-nun-thick Y-cut quartz crystal (a piece of quartz plate whose plane is perpendicular to the crystalline y-axis and the thickness of the plate is $3 \mathrm{~mm}$. and $\mathrm{d}_{11}=0.3 \mathrm{pm} / \mathrm{V}$ ) was used as a reference for determining the relative intensities of the $\mathrm{SH}$ signals generated from the samples. The Maker Fringe pattern was obtained by measuring the SHG signal at $0.5^{\circ}$ intervals using a rotation stage. $\mathrm{SHG}$ coefficients $\left(d_{33}\right)$ were derived from the analysis of measured Maker-fringes. ${ }^{1}$

2,4-(2'-Vinyloxyethoxy)benzaldehyde (1). 2.4-Dihydroxybenzaldehỵde ( 13.8 g. $0.10 \mathrm{~mol})$. anhỵdrous potassium carbonate $(82.9 \mathrm{~g}, 0.60 \mathrm{~mol})$ and 2 -iodoethyl vinyl ether $(49.5 \mathrm{~g}$, $0.25 \mathrm{~mol}$ ) were dissolved in $400 \mathrm{~mL}$ of dry DMF under nitrogen. The nixture was refluxed in an oil bath kept at $80^{\circ} \mathrm{C}$ for $15 \mathrm{hr}$ under nitrogen. The resulting solution was cooled to room temperature, diluted with $300 \mathrm{~mL}$ of water. and extracted 
with $300 \mathrm{~mL}$ of diethyl ether three times. The organic layer was washed with saturated aqueous sodium chloride solution. and dried with anhydrous magnesium sulfate. Rotary evaporation of diethyl ether gave crude product, which was recrystallized from 1-butanol yielded $25.0 \mathrm{~g}$ (yield $90 \%$ ) of pure product 1. M.p. $68-69{ }^{\circ} \mathrm{C}$. ${ }^{1} \mathrm{H}$ NMR (acetone- $\left.d_{6}\right) \delta 4.03-4.35$ (m. 12H. $\left.2 \mathrm{CH}_{2}=.2-\mathrm{O}-\mathrm{CH}_{2}-\mathrm{CH}_{2}-\mathrm{O}-\right)$. 6.50-6.62 (m. 4H. 2 $=\mathrm{CH}-\mathrm{O}-$, aromatic). 7.82-7.86 (d. $1 \mathrm{H}$, aromatic). $10.35(\mathrm{~s}, 1 \mathrm{H}$, -CHO). IR (KBr) $3100,3082(\mathrm{w} .=\mathrm{C}-\mathrm{H}), 2954,2875(\mathrm{~m}$. C-H) 1674 (vs. $\mathrm{C}=\mathrm{O}$ ), 1615 (vs. $\mathrm{C}=\mathrm{C}$ ). 1575 (s. C=C) $\mathrm{cm}^{-1}$.

Methyl 2,4-Di-(2'-vinyloxyethoxy)benzylidenecyanoacetate (2). Piperidine $(0.13 \mathrm{~g} .1 .5 \mathrm{mmol})$ was added to a solution of 2,4-di-(2'-vinyloxyethoxy)benzaldehyde 1 (8.35 g. 30 mmol) and methyl cyanoacetate $(3.27 \mathrm{~g} .33 \mathrm{mmol})$ in $200 \mathrm{~mL}$ of $n$-butanol with stirring at 0 " $\mathrm{C}$ under nitrogen. After stirring for 3 h at $0^{\circ} \mathrm{C}$, the reaction mixture was cooled to $-10^{\circ} \mathrm{C}$ for crystallization. The product was filtered and washed successively with cold $n$-butanol $(80 \mathrm{~mL})$, water $(30 \mathrm{~mL})$. and cold $n$-butanol $(20 \mathrm{~mL})$. The obtained pale yellow product was recry stallized from 1-butanol to give $9.49 \mathrm{~g}$ ( $88 \%$ yield) of 2 . M.p. $96-97{ }^{\circ} \mathrm{C} .{ }^{1} \mathrm{H}$ NMR $\left(\mathrm{CDCl}_{3}\right)$ ò 3.89 (s. 3H. $-\mathrm{CO}_{2} \mathrm{CH}_{3}$ ). $4.02-4.34\left(\mathrm{~m} .12 \mathrm{H}, 2 \mathrm{CH}_{2}=, 2-\mathrm{O}-\mathrm{CH}_{2}-\mathrm{CH}_{2}-\mathrm{O}-\right), 6.46-6.65$ (m, 4 H. 2 =CH-O-. aromatic), 8.36-8.428(d. 1H, aromatic). 8.70 (s. IH. aromatic). IR (KBr) $3044(\mathrm{w},=\mathrm{C}-\mathrm{H}) .2954 .2943$ (m, C-H), 2222 (s, CN). 1705 (vs. C=O). 1622.1611. 1594. 1583 (vs, $\mathrm{C}=\mathrm{C}$ ) $\mathrm{cm}^{-1}$. Anal. Calcd for $\mathrm{C}_{15} \mathrm{H}_{21} \mathrm{NO}_{6}: \mathrm{C}, 63.50$ : H. 5.89: N. 3.90. Found: C. 63.62: H. 5.82: N. 3.83 .

2,4-Di-(2'-hydnoxyethoxy) benzylidenecyanoacetate (3). Aqueous hydrochloric acid (1.5 M.30 mL) was slow ly added to a solution of methyl 2.4-di-(2'-vinyloxyethoxy)benzylidenecyanoacetate (2) $(9.3+\mathrm{g} .0 .026 \mathrm{~mol})$ in $60 \mathrm{~mL}$ of dry THF with stirring under nitrogen at $0^{\circ} \mathrm{C}$. The mixture was stirred at $80^{\circ} \mathrm{C}$ for $8 \mathrm{~h}$ under nitrogen. The resulting solution was extracted with diethyl ether $(80 \mathrm{~mL})$ three times. The organic layer was washed successively with saturated sodium chloride. sodium hydrogen carbonate and water. followed by drỵing with anhydrous magnesium sulfate. Rotary evaporation of diethyl ether gave cnide product. The obtained pale yellow product was recrystallized from ethyl acetate to give $6.87 \mathrm{~g}$ (86\% yield) of 3. M.p. $168-170{ }^{\circ} \mathrm{C}{ }^{1} \mathrm{H} \mathrm{NMR}\left(\mathrm{CDCl}_{3}\right) \delta 2.78-$ $2.85(\mathrm{~m} .2 \mathrm{H} .-\mathrm{OH}) .3 .84-3.89$ (s. $\left.3 \mathrm{H} \mathrm{CO}_{2} \mathrm{CH}_{3}\right) .3 .90-3.98(\mathrm{~m}$. 2H. $\left.-\mathrm{CH}_{2}-\mathrm{OH}\right) \cdot 4.16-4.28\left(\mathrm{~m} .2 \mathrm{H}\right.$. $\left.-\mathrm{O}-\mathrm{CH}_{2}-\right)$. 6.72-6.76 (m. $2 \mathrm{H}$. aromatic) 8.32-8.36 (d. $\mathrm{IH}$. aromatic) $8.70-8.72(\mathrm{~d} . \mathrm{IH}$. -Ph-CH=). IR (KBr) 3514,3435 (s. O-H), 3044 (w; =C-H). 2955 (m, C-H), 2216 (m. CN), 1709 (vs. C=O). 1612.1578 (vs. $\mathrm{C}=\mathrm{C}$ ) $\mathrm{cm}^{-1}$. Anal. Calcd for $\mathrm{C}_{15} \mathrm{H}_{1}: \mathrm{NO}_{6}$ : C. 58.63: H. 5.57: $\mathrm{N}, 4.56$. Found: $\mathrm{C}, 58.76 \mathrm{H}, 5.63: \mathrm{N}, 4.65$.

Synthesis of Polyester4. A representative polycondensation procedure was as follows: Terephthaloyl chloride $(2.03 \mathrm{~g}$. $0.01 \mathrm{~mol})$ and diol $3(3.07 \mathrm{~g} .0 .01 \mathrm{~mol})$ were dissolved in 30
$\mathrm{nL}$ of anhydrous pyridine under nitrogen. The resulting solution was refluxed in an oil bath kept at $80^{\circ} \mathrm{C}$ under a nitrogen atmosphere. After heating $10 \mathrm{~h}$ with stirring the resulting poly merization solution was poured into $400 \mathrm{~mL}$ of methanol. The precipitated polymer was collected and reprecipitated from DMSO into methanol. The polymer was further purified by extraction in a Soxhlet extractor with diethyl ether and dried under vacuunt. yielding $3.98 \mathrm{~g}$ ( $91 \%$ yield) of polymer 4 . Inherent viscosity $\left(\eta_{\text {inh }}\right)=0.30 \mathrm{dL} \mathrm{g}^{-1}\left(\mathrm{c}=0.5 \mathrm{~g} \mathrm{dL}^{-1}\right.$ in DMSO at $25^{\circ} \mathrm{C}$ ). ${ }^{1} \mathrm{H}$ NMR (DMSO-d $\left.d_{6}\right) \delta 3.65-3.73$ (s. $3 \mathrm{H}$. $\left.-\mathrm{COOCH}_{3}\right), 4.55$ (s. +H. 2 -PhOCH$=$ -, 4.68 (s. + H. 2 COO$\left.\mathrm{CH}_{2}\right), 6.75-6.95$ (m. 2H, aromatic). 8.05 (s. $4 \mathrm{H}$. aromatic). 8.18 (d. $1 \mathrm{H}$, aromatic). 8.54 (s. $1 \mathrm{H}$. aromatic). IR ( $\mathrm{KBr}) 2955$ (m. C-H) 2220 (s. CN). 1722 (vs. C=O). 1586 (s. C=C) $\mathrm{cm}^{.1}$. Anal. Calcd for $\left(\mathrm{C}_{22} \mathrm{H}_{19} \mathrm{NO}_{3}\right)_{13}: \mathrm{C} .63 .16: \mathrm{H}, 4.37: \mathrm{N} .3 .20$. Found: C. 63.24: H, 4.43: N, 3.17 .

Aclonowledgments. This work was supported by Korea Research Foundation Grant funded by the Korean Government (KRF-2007-313-C00498).

\section{References}

1. Cho, M. J.; Kim, I. Y.; Kim, J. H.; Lee, S. H.; Dalton, L. R: Choi, D. H. Bull Kowean Chem. Soc. 2005, 26,77.

2. Lee, C.; Park, S.-K.; Yang, M.; Lee, N.-S.; Kim, N. J. Butl. Korean Chem. Soc. 2007, 28, 447.

3. Kim, T.-D; Kang, J-W.: Luo, J.: Jang, S.-H.; Ka, J-W.: Tucker, N.: Benledict, J. B.; Dalton, L. R:- Gray, T.; Ovener, R. M.; Park, D. H.: Herman, W. N.; Jen, A. K.Y. J. Am. Chem. Soc. 2007. 129, 488 .

4. Yoon, Z. S.: Easwaramoorthi, S.: Kim, D. Bull Korean Chem. Soc. 2008, 29, 197

5. Han, K. S.; Park, S. K; Shim, S. Y ; Jahng, W. S ; Kim, N. I. Bull. Korean Chem. Soc. 1998, 19, 1165

6. Han, K. S.; Park, S. K.; Shim, S. Y.; Lee, Y. S.; Jahng, W. S.; Kim. N. J. Bull. Korean Chem. Soc. 1998, 19, 1168.

7. Cho, B. R.; Kim, Y. H.: Son, K. W: Khalil, C.: Kim, Y. H.; Jeon, S.-J. Bull. Korean Chemt. Sac 2002, 23, 1253.

8. Moon, I. R.: Kim, B. S.: Kim, T.-H. Bull. Korean Chem. Soc. 2006, 27,981,

9. Kim, M. H.; Tin, J.-I; Lee, C. J.; Kim, N. T.; Park, C. H. Bull. Korean Chem. Soc. 2002, 23,964.

10. Lee, J.-H; Lee, K.-S. Bull. Korew Chem. Soc, $2000,21,847$.

11. Lee, S.-H.; Kim, Y.-K.; Won, Y.-H. Hacromolecules 1999, 32 , 342

12. Lee, J-Y.; Jung, W.-T; Lee, W.-J. Potwn. Int $\mathbf{2 0 0 6}, 55,248$.

13. Lee, J.-Y.: Kim, I.-H.: Iung, W.-T. Bull Korean Chem. Soc. $\mathbf{2 0 0 7}, 28,329$

14. Lee, I.-Y; Kim, J.-H.; Won, D.-S.; Jang, H.-N. Bull. Kolean Chent Soc. 2007, 28, 1433.

15. Jang, H.-N.; Lee, T.-Y. Bull. Korean Chem. Soc 2008, $29,933$.

16. Cisneros, J. I. Appl. Opt. 1998, 37, 3262 .

17. Herman, W. N.; Hay den, L. M. J. Opt. Soc. Am. B 1995, $12,416$. 